\title{
A Nanomesh Scaffold for Supramolecular Nanowire Optoelectronic Devices
}

Lei Zhang ${ }^{1}$, Xiaolan Zhong ${ }^{1}$, Egon Pavlica ${ }^{2}$, Songlin $\mathrm{Li}^{1}$, Alexander Klekachev ${ }^{1}$, Gvido Bratina $^{2}$, Thomas W. Ebbesen ${ }^{1}$, Emanuele Orgiu ${ }^{1}$ and Paolo Samori ${ }^{1 *}$

${ }^{1}$ ISIS \& icFRC, University of Strasbourg and CNRS, 8 allée Gaspard Monge, Strasbourg, 67000, France

${ }^{2}$ Laboratory of Organic Matter Physics, Vipavska 11c, SI-5270 Ajdovščina, University of Nova Gorica, Slovenia

Emails: samori@unistra.fr, orgiu@unistra.fr

\begin{abstract}
Supramolecular organic nanowires are ideal nanostructures for optoelectronics because they exhibit both efficient exciton generation, by virtue of their high absorption coefficient, and remarkable light sensitivity, by virtue of a small number of grain boundaries and high surface-to-volume ratio. To harvest photocurrent directly from supramolecular nanowires it is necessary to wire them up with nanoelectrodes possessing different work functions. However, devising strategies that can connect multiple nanowires at the same time has been challenging. Here we report a general approach to simultaneously integrate hundreds supramolecular nanowires of $\mathrm{N}, \mathrm{N}^{\prime}$-Dioctyl-3,4,9,10-perylenedicarboximide (PTCDI-C8) in a hexagonal nanomesh scaffold featuring asymmetric nanoelectrodes. Optimized PTCDI-C8 nanowires photovoltaic devices exhibit a signal-to-noise ratio approaching $10^{7}$, a photoresponse time as fast as $10 \mathrm{~ns}$ and an external quantum efficiency $>55 \%$. This nanomesh scaffold can also be used to investigate the fundamental mechanism of photoelectrical conversion in other low-dimensional semiconducting nanostructures.
\end{abstract}

Semiconducting supramolecular nanowires are versatile nanostructures that combine the advantages of soft materials like polymers in terms of flexibility and low-cost solution-processability with the physical and chemical characteristics of organic crystals. ${ }^{1-4}$ Along these nanowires, photo-generated charge carriers can be transported efficiently to reach the electrodes by virtue of optimal $\pi$-electron overlapping. ${ }^{5-7}$ Supramolecular nanowires are also particularly suitable for exciton dissociation because their high surface-to-bulk ratio 
minimizes the diffusion length from the bulk to the interface where exciton separation typically occurs. ${ }^{8-10}$ Recently, long-range exciton transport, up to a few microns, has been reported. ${ }^{11}$ Other advantages for photonic applications include lower interface reflection loss compared to thin-film, ${ }^{10,12}$ and the possibility to tune the optoelectronic properties by rational molecular design and aggregation. ${ }^{13-16}$ All these characteristics make supramolecular nanowires ideal active components in optoelectronic devices. In addition, these devices represent a perfect platform for studying how the order at the supramolecular level affects photoelectrical conversion. ${ }^{17,18}$

So far, a variety of supramolecular nanowires have been synthesized and investigated. ${ }^{19-24}$ Some of them have been used in organic field-effect transistors and phototransistors showing promising optoelectronic properties. ${ }^{25-27}$ However, photovoltaic devices based solely on organic nanowires are challenging to fabricate, because it is hard to control the interface between the nanowire and the electrodes, where the dissociated exciton are to be collected. ${ }^{28,29}$ In order to efficiently harvest photocurrent from nanowires, one of the prerequisites is to interface them with an external circuit possessing asymmetric nanoelectrodes. At the same time, the nanoelectrodes should also cover a large area so as to incorporate as many nanowires as possible. Since low-cost production represents one of the advantages of this technology, the nanoelectrodes should also be easy to fabricate. Although these requirements could be satisfied in a thin-film vertical diode configuration, such approach is not applicable to neat one-dimensional semiconductors because nanowires alone would not form an even and pinhole-free layer to sustain the top electrode without the addition of a strengthening polymer layer. ${ }^{8}$ Furthermore, making electrical contact with bottom-up nanostructures is still a long-standing research topic in supramolecular electronics. ${ }^{30-32}$

Here we report a vertical-channel nanomesh scaffold that supports and connects supramolecular nanowire between two nanoelectrodes with different work functions. Patterned by nanospheres lithography, the scaffold comprises millions of hole-shaped nanoelectrodes in a hexagonal array with a channel length below $100 \mathrm{~nm}$. The height difference between top and bottom electrodes enables zone-selective modification with a series of organic/polymeric semiconductors to further tune the asymmetry between the 
electrodes. We have focused our attention to N,N'-Dioctyl-3,4,9,10-perylenedicarboximide (PTCDI-C8) nanowires as model system for optoelectronics. PTCDI-C8 is a commercially available n-type organic semiconductor that self-assembles into supramolecular nanowires through solvent-induced-precipitation. ${ }^{14,21,25}$ When asymmetrically connected to nanoelectrodes, PTCDI-C8 nanowires exhibit well-defined photovoltaic effect under monochromatic visible light. In particular, the resulting photonic device shows high external quantum efficiency when in resonance, ultrafast photoresponse and high signal-to-noise ratio because of an efficient charge and exciton transport. ${ }^{25,33}$

\section{RESULTS AND DISCUSSION}

\section{Fabrication of honeycomb-shaped asymmetric nanoelectrodes}

Nanospheres lithography refers to a patterning approach that utilizes hexagonal close-packed (hcp) nanospheres monolayer as mask. ${ }^{34}$ By combining additive- and subtractive-process, nanospheres lithography offers a versatile method to fabricate large-area and periodic nanostructures with different materials like metals, graphene, semiconductors etc. ${ }^{35-37}$ The fabrication procedure starts with the deposition of a polystyrene (PS) nanospheres hcp-monolayer (Fig. 1a). Then oxygen plasma treatment is applied to widen the gap between adjacent PS nanospheres (Fig. S1 in the Supporting Information). Au is then deposited by high-vacuum thermal evaporation followed by a lift-off step (Fig. S2). Thereafter, the gold nanomesh serves as a mask to remove $\mathrm{SiO}_{2}$ dielectric layer by reactive ion etching. The exposed surface of heavily n-doped Si was designed to act as the counter-electrode. Therefore, the inter-electrode distance in our device is determined by the $\mathrm{SiO}_{2}$ thickness $(90 \mathrm{~nm}$ in our case). To estimate the yield of fabrication process, we carried out a large device statistics and found that $83 \%$ out of the total 160 devices exhibited leakage current below $1 \mathrm{nA}$ at $\pm 1.5 \mathrm{~V}$ bias voltage (Fig. S3; Surface area: $0.07 \mathrm{~cm}^{2}$ ).

Significantly, our approach is extremely versatile, e.g. it is possible to make further modifications inside the nanohole (Fig. 1b). ${ }^{38}$ Firstly, a thin layer of semiconducting p-type polymer like poly(3-hexylthiophene) (P3HT) was spin-coated without altering the nanomesh morphology. This process is referred to as "conformal spin-coating", which is realized by 
depositing a very thin film onto a relatively rough surface containing nanostructures. In contrast, if the spin-coated film were much thicker, the nanostructured surface would be smoothened as shown in the second step of Fig. 1b. Here the substrate flattens by poly(4-vinylphenol) (PVP) sacrificial layer was then transferred into an oxygen plasma chamber to "burn off" redundant PVP film. By controlling the etching dose, only P3HT modification layer inside nanoholes is left since PVP could be washed away by ethanol thereafter. For each step of the procedure depicted in Figure 1b, the change in morphology has been characterized by atomic force microscope (AFM) (see Fig. S4).

Figure 1c and $\mathrm{d}$ show the photograph images of fully covered PS nanospheres monolayer with $1100 \mathrm{~nm}$ sphere diameter and the resulting nanomesh scaffold, respectively. The polychromatic shades originate from light dispersion effects of nanosphere- or hole-array with period comparable to visible light wavelength. Figure $1 \mathrm{e}$ and $1 \mathrm{f}$ portray the topographical AFM images of samples displayed in Fig. 1c and 1d, respectively. As suggested by the colourful appearance in photograph images, the PS nanospheres monolayer and honeycomb-like hole-array scaffold show high degree of periodicity. The SEM analysis performed at a tilt angle of $54^{\circ}$ (see Fig. $1 \mathrm{~g}$ and $1 \mathrm{~h}$ ) further confirm that $i$ ) the side walls are vertical and sharp, and ii) the gold nanomesh stays on top of dielectric layer without collapsing. The latter observation is in accordance with the low leakage current level measured as reported in Fig. S3.

\section{Optoelectronic properties of nanowires on bare nano-scaffold}

Our geometry was used to incorporate large-area and full-coverage nanowires films produced through hierarchical assembly (Fig. 1i). In particular, PTCDI-C8 molecules were self-assembled into supramolecular nanowires by solvent-induced-precipitation. Then, the obtained supramolecular nanowires were organized into quasi-monolayer thick films floating at the water/air interface (see method section and Fig. S5). ${ }^{39}$ Before transferring them onto the nanomesh scaffold, $50 \mu \mathrm{L}$ of PVP solution (ethyl acetate; $100 \mathrm{mg} / \mathrm{ml}$ ) was dropped onto the water surface to compress the floating supramolecular nanowires into uniform films. Compared to spin-coating, this assembling approach has two advantages: $i$ ) the supramolecular nanowires can be even distributed on surfaces, as confirmed by fluorescence 
microscopy (Fig. S5), and ii) $100 \%$ of material can be deposited at surfaces, without any waste. Figure 2a displays the SEM images of full-coverage supramolecular nanowires on top of a nanomesh scaffold. It reveals that almost every nanohole is accommodating nanowires as evidenced further in the zoom-in in Fig. $2 b$ and 2c.

After PTCDI-C8 nanowires deposition and thermal annealing inside glovebox, we successfully observed photovoltaic effect which could be attributed to the light absorption by PTCDI-C8 supramolecular nanowires. Figure $2 \mathrm{~d}$ portrays exemplary $I-V$ curves in dark and under monochromatic light $\left(470 \mathrm{~nm} ; 212.9 \mathrm{~mW} / \mathrm{cm}^{2}\right)$. The inset in Fig. $2 \mathrm{~d}$ shows the molecular structure of PTCDI-C8. During the measurement, Si is grounded to $0 \mathrm{~V}$, so we can define the gold nanomesh as cathode according to the photocurrent direction. Since PTCDI-C8 is an n-type semiconductor, the excitons should separate at nanowires/Si interface. Then photo-generated charges are efficiently transported though the nanowires and are collected at the gold cathode. Thin film X-ray diffraction (XRD) analysis was also carried out to shed light on the molecular stacking in these solvent-induced-precipitation derived supramolecular nanowires (Fig. S5). While distinct diffraction peaks confirm the crystalline nature of the nanowires, the peaks position suggests that disc-like PTCDI-C8 molecules exhibit slipped $\pi-\pi$ stacking along [100] direction corresponding to the long axis of the nanowire, thereby favouring efficient in-plane charge carrier transport. ${ }^{14}$

To investigate how the geometry parameters influence the total photocurrent, we adjusted the size of nanowires by mixing PTCDI-C8 solution with a series of alcohol from methanol to 1-hexanol as non-solvent. Generally, high boiling point alcohols, i.e. with increasing number of carbon atoms in the main chain, enable slow molecular diffusion thereby providing longer times for PTCDI-C8 to self-assemble and form supramolecular nanowires (Fig. S6). As a result, the size of the nanowires increases monotonically with the number of carbon atoms in the alcohol molecule of the non-solvent (Fig. S7). The photo response spectrum and photocurrent dependence on different nanowires size are shown in Fig. S8 and Fig. 2e. Interestingly, the photocurrent of supramolecular nanowires from different non-solvents peaks when ethanol is used (Fig. 2e). On the one hand, bigger nanowires could be beneficial thanks to the enhanced light absorption from thicker active layer. On the other hand, thicker and wider nanowires possess increased rigidity. When the rigidity is larger, e.g. 
for the case of 1-butanol, 1-pentanol and 1-hexanol, nanowires will bridge the two sides of the hole without necessarily touching the underlying Si electrode (Fig. S7). In this case, both the photocurrent and dark current will decrease significantly because of the poor contact between nanowires and $\mathrm{Si}$.

In order to verify this hypothesis, we have also deposited identical supramolecular nanowires film onto different nanomesh scaffold patterned by nanospheres with diameter of $480 \mathrm{~nm}, 600 \mathrm{~nm}, 800 \mathrm{~nm}$ and $1100 \mathrm{~nm}$. The $I_{\mathrm{SC}}$ and $V_{\mathrm{OC}}$ values are summarized in Table 1. By keeping all the remaining geometrical parameters unchanged, we have found that larger nanoholes provide greater space for nanowires to bend and ultimately make a suitable physical (i.e. electrical) contact with Si (Fig. S9). In accordance to the prediction that the photocurrent should be proportional to the area of nanowires/Si interface, we found that $I_{\mathrm{SC}}$ increases when larger diameter patterns are used (Table 1). Hence, the roughness of nanomesh scaffold and nanowires conformity jointly determine the effective area of nanowires/Si interface, which should be linearly associated with the overall photocurrent. The geometry parameter has been fully optimized to target larger nanowire/Si interface for higher photocurrents. It should be noted that the gold nanomesh forms a plasmonic structure that can also potentially act like an antenna to further boost the absorption if properly optimized. ${ }^{40}$ No clear evidence for this effect could be detected in the present study probably due to the fact that the organic nanowire absorbs at the interband transition of Au. However, for nanowires absorbing at longer wavelengths the nanomesh could favour both the electronic and the photonic functionalities.

\section{Exciton separation enhancement with P3HT-modified anode}

When heavily-doped Si is contacted with organic semiconductors, it always behaves as hole injecting electrode regardless if the employed silicon is $n$ - or p-type doped. ${ }^{41}$ Here we have indeed observed hole collection from PTCDI-C8 nanowires by n-Si (Fig. 2d). However, the best photoresponsivity amounted to $10-20 \mu \mathrm{A} / \mathrm{W}$. The Fermi energy of n-doped Si lies approximately in the center of PTCDI-C8 bandgap thus enabling both electron and hole collection process at such electrode. In order to enhance hole-selectivity and suppress non-radiative recombination at $\mathrm{Si} /$ nanowires interface, the $\mathrm{Si}$ could be modified with a layer 
of p-type semiconducting polymer, inhibiting electron transfer from the PTCDI-C8 to the Si electrode by forming p-n junction with PTCDI-C8 supramolecular nanowires.

According to the method described in Fig. 1b, a ca. $5 \mathrm{~nm}$ thick layer of P3HT was deposited to selectively modify the Si surface and to facilitate hole transport across a p-type semiconductor. Such interface tailoring resulted in a significant $I_{\mathrm{SC}}$ enhancement up to 500-fold after nanowires deposition along with a $V_{\mathrm{OC}}$ improvement from $0.18 \mathrm{~V}$ to $0.30 \mathrm{~V}$. As a photodetector, the signal-to-noise ratio of our PTCDI-C8 supramolecular nanowires devices increased from $10^{4}-10^{5}$ to $10^{7}$ in photovoltaic mode as a result of the anode modification. The P3HT interlayer could also reduce the dark current to $\sim 10 \%$ of its initial level when $1.5 \mathrm{~V}$ working voltage was applied, so that the signal-to-noise in photoconduction mode improved significantly of three orders of magnitude, i.e. from 10 to $10^{4}$ (Fig. 3a).

To quantify more accurately the efficiency of the photovoltaic process in supramolecular nanowires, we acquired SEM images of the device and counted the number of nanowires inside single nanoholes (Fig. S10). The analysis displayed in Fig. S11 reveals that $4.05 \%$ of the whole device surface is occupied by the PTCDI-C8/P3HT interface. Furthermore, we also fabricated and tested supramolecular nanowires device with smaller surface area $\left(28 \times 28 \mu \mathrm{m}^{2}\right)$, which could be viewed within a single AFM image, making it possible to quantify more precisely the photovoltaic performances with respect to those measured in large-area devices (Fig. 3b; Fig. S11). In the small device, the PTCDI-C8/P3HT interface was estimated to occupy $9.08 \%$ of the whole device surface. Calibrated by real p-n interface area, the peak external quantum efficiency (EQE) could reach $47 \%$ when illuminated through a 500-nm green light for the device shown in Fig. 3b. A similar EQE value of $55.4 \%$ is achieved for a large-area device under the same illumination condition (Fig. 3c). For both large and small area supramolecular nanowires devices, the EQE, photoresponsivity $(R)$ and PCE showed a well-distinct wavelength dependence with the best results obtained at PTCDI-C8 nanowires absorption peak (Fig. 3c; Fig. S12 and Fig. S13). Because the average thickness of the supramolecular nanowires employed here is only $\sim 45$ $\mathrm{nm}$ to maintain the required flexibility, the insufficient light absorption represents, together with low p-n junction occupation, the main limiting factors for further performance improvement. 
As illustrated in Fig. 3d and related inset, the current in the photoconduction region $(1.5 \mathrm{~V})$ manifested better linear relationship with rising incident light power density than in the photovoltaic region $(0 \mathrm{~V})$. In other words, the photocurrent saturation effect could be somewhat limited when the device is biased, because the applied electric field could help to remove accumulated electrons at the interface in terms of drift current. Additionally, the external voltage bias would also enhance the photo responsivity, i.e. photocurrent, for several times (Fig. 3d). For example, the photo responsivity spectrum of small surface supramolecular nanowires device $\left(28 \times 28 \mu^{2}\right)$ was investigated by varying the working voltage from $0 \mathrm{~V}$ to $1.5 \mathrm{~V}$ and the data are summarized into a colour-filling contour image (Fig. 3e). The maximum photo responsivity $(R)$ in photovoltaic mode is $\sim 196 \mathrm{~mA} / \mathrm{W}$ after calibration and it is further improved to $1.2 \mathrm{~A} / \mathrm{W}$ when $1.5 \mathrm{~V}$ voltage bias is applied (photoconduction mode). Such a responsivity value is among the best results reported for organic photodiodes. ${ }^{42}$ Our PTCDI-C8 supramolecular nanowires photovoltaic device could also be operated under very low light power density. As shown in Fig. S14, the current still displays a 10 -fold increase when irradiated by $0.018 \mu \mathrm{W} / \mathrm{cm}^{2}$ green light.

The short inter-electrodic distance and good charge transport ability make this supramolecular nanowires photodetector switch faster than previously reported phototransistors with longer channel lengths. ${ }^{42,43}$ Figure $3 \mathrm{f}$ shows that the photocurrent in the supramolecular nanowires photonic device starts to rise immediately after laser irradiation and the current peaks $\sim 10 \mathrm{~ns}$ after the laser pulse hits the sample in the case of a biased device (photoconduction mode, $\mathrm{V}=1.5 \mathrm{~V}$ ). Similar response time in photoconduction mode has also been demonstrated for bare nanomesh scaffold without P3HT modification (Figure S16). As for the photovoltaic mode, the device switches on a slightly longer times with photocurrent peak at $\sim 20$ ns because of lack of an external driving voltage. Such ultrafast photoresponse time within tens of ns proves that our supramolecular nanowires photonic devices are suitable for operating at high frequency.

\section{Tuning of photovoltaic properties by hole transport layers}

In this study, the possibility of modifying the anode with different polymers makes it possible to unravel the origin of $V_{\mathrm{OC}}$ in photovoltaic process, which could be helpful to 
understand the working principle of supramolecular nanowires photonic devices. Here we investigated how the energy level alignment of p-n junction influences the $V_{\mathrm{OC}}$ by changing different hole transport layers including P3HT, IIDDT-C3 and F8T2. ${ }^{44,45}$ The UV-Vis absorption spectra and HOMO-LUMO energy levels are summarized in Fig. 4a. From Figure $4 \mathrm{~b}$ and Table 2, we discovered that larger HOMO energy difference $(\triangle \mathrm{HOMO})$ between donor and acceptor leads to enhanced $I_{\mathrm{SC}}$ but smaller $V_{\mathrm{OC}}$. Different from P3HT/PCBM system, here the main driving force for excitons dissociation at donor/acceptor interface is $\triangle \mathrm{HOMO}$ rather than $\triangle \mathrm{LUMO}^{46}$ Although large $\triangle \mathrm{HOMO}$ should lead to a more efficient exciton separation, a large $\triangle \mathrm{HOMO}$ would also represent a larger energy loss at the interface. In organic solar cells, such kind of energy loss in terms of heat would typically sacrifice $V_{\mathrm{OC}}$ to a certain degree. The latter consideration supports the experimental evidence that F8T2-modified devices exhibited larger $V_{\mathrm{OC}}$ than P3HT-modified devices, and IIDDT-C3/PTCDI-C8 interface would reach the largest $V_{\mathrm{OC}}$ (Fig. 4b; Table 2 and Table S1). The relationship between FF and the conductivity of each modification layer is discussed in Fig. S17 and S18.

Fig. $4 \mathrm{c}$ shows the photo responsivity $(R)$ spectra recorded on the above mentioned modification layers and bare device without modification. In view of the reduced thickness which is less than the typical exciton diffusion length for polymers $\left(L_{\mathrm{ex}} \sim 10 \mathrm{~nm}\right),{ }^{47}$ the efficiency of exciton transport and separation in the hole transporting layer should be high. Since the photocurrent is apparently determined by the absorption superposition of PTCDT-C8 nanowires and the modification layer, we can deduce that the exciton diffusion length in PTCDI-C8 supramolecular nanowires should be larger than or at least comparable with the nanowire thickness of $\sim 45 \mathrm{~nm}$, otherwise the modification layer would contribute more significantly to the photocurrent than in Fig. 4c. The much longer $L_{\mathrm{ex}}$ than amorphous polymers originates from the ordered molecular stacking in supramolecular nanowires. ${ }^{48,49}$ Delocalized excitons and their efficient transport in PTCDI-based dye aggregates was reported by the aid of transient fluorescence analysis, ${ }^{50}$ which is consistent with our electronic device measurement. 


\section{CONCLUSIONS}

In summary, we have reported direct light energy harvesting from supramolecular nanowires by connecting them to a nanomesh scaffold which was fabricated at macroscopic, centimetre scale. Our device exhibits numerous advantageous features. First, the polymer/nanowire p-n junction features a fast photoresponse owing to the reduced anode-cathode distance. Second, the possibility to chemically modify anode and cathode separately enables the realization of tailored interfaces to replace conventional $\mathrm{Ca}$ and $\mathrm{Al}$ cathodes, and makes it unnecessary to use a transparent electrode like ITO. Third, such device geometry guarantees that the current will flow only across the p-n junction area therefore not following side pathways that generate shunt parasitic connections. Currently, a model PTCDI-C8 nanowire photovoltaic device shows an external quantum efficiency $>55 \%$, a signal-to-noise of $10^{7}$ and a response time as fast at $10 \mathrm{~ns}$. In the future, further device optimization can be achieved in various fronts. For example, thin films of polymer insulators on plastic substrates could be used to endow flexibility and further cost reduction. Thinner dielectric layers and nanoholes with tailored size as well as more flexible nanowires could increase the overall photocurrent by maximizing the contact area between the nanowire and the anode. Another possible approach to improve the contact area is to flatten and smoothen the nanomesh scaffold with semiconducting organic crystals by selectively crystal seeding and growing inside nanoholes. Significantly, other photoactive low-dimensional architectures such as inorganic nanowires or organic dye aggregates could be integrated in our device to investigate their inherent photonic properties, as long as the requirements of high absorption coefficient, good wire conductivity and conformity to the nanomesh scaffold are fulfilled. Furthermore, our vertical-channel device configuration can be exploited for fundamental studies in other organic nano-structured optoelectronic devices, such as light-emitting diodes, or even spintronics like spin-valves based on nanowires, because for these devices the combination of asymmetric electrodes with ad-hoc work-function, desirable spin transport, nanoscale controlled channel length and maximal density of active nanostructures are vital. 


\section{References}

1. Hoeben, F.J.M., Jonkheijm, P., Meijer, E.W. \& Schenning, A. About supramolecular assemblies of pi-conjugated systems. Chem. Rev. 105, 1491-1546 (2005).

2. Lee, C.C., Grenier, C., Meijer, E.W. \& Schenning, A. Preparation and characterization of helical self-assembled nanofibers. Chem. Soc. Rev. 38, 671-683 (2009).

3. Chen, Z.J., Lohr, A., Saha-Moller, C.R. \& Wurthner, F. Self-assembled pi-stacks of functional dyes in solution: structural and thermodynamic features. Chem. Soc. Rev. 38, 564-584 (2009).

4. Jain, A. \& George, S.J. New directions in supramolecular electronics. Mater. Today 18, 206-214 (2015).

5. Jiang, L., Fu, Y., Li, H. \& Hu, W. Single-crystalline, size, and orientation controllable nanowires and ultralong microwires of organic semiconductor with strong photoswitching property. J. Am. Chem. Soc. 130, 3937-3941 (2008).

6. Marty, R. et al. Hierarchically structured microfibers of "single stack" perylene bisimide and quaterthiophene nanowires. Acs Nano 7, 8498-8508 (2013).

7. Wei, L., Yao, J.N. \& Fu, H.B. Solvent-assisted self-assembly of fullerene into single-crystal ultrathin microribbons as highly sensitive UV-visible photodetectors. Acs Nano 7, 7573-7582 (2013).

8. Wicklein, A., Ghosh, S., Sommer, M., Würthner, F. \& Thelakkat, M. Self-assembly of semiconductor organogelator nanowires for photoinduced charge separation. ACS Nano 3, 1107-1114 (2009).

9. Che, Y. et al. Interfacial engineering of organic nanofibril heterojunctions into highly photoconductive materials. J. Am. Chem. Soc. 133, 1087-1091 (2011).

10. Tian, B.Z. et al. Coaxial silicon nanowires as solar cells and nanoelectronic power sources. Nature 449, 885-890 (2007).

11. Haedler, A.T. et al. Long-range energy transport in single supramolecular nanofibres at room temperature. Nature 523, 196-199 (2015).

12. Garnett, E. \& Yang, P.D. Light trapping in silicon nanowire solar cells. Nano Lett. 10, 1082-1087 (2010). 
13. Briseno, A.L. et al. Fabrication of field-effect transistors from hexathiapentacene single-crystal nanowires. Nano Lett. 7, 668-675 (2007).

14. Briseno, A.L. et al. Perylenediimide nanowires and their use in fabricating field-effect transistors and complementary inverters. Nano Lett. 7, 2847-2853 (2007).

15. An, B.K., Gierschner, J. \& Park, S.Y. pi-Conjugated cyanostilbene derivatives: a unique self-assembly motif for molecular nanostructures with enhanced emission and transport. Acc. Chem. Res. 45, 544-554 (2012).

16. Walker, B.J., Dorn, A., Bulovic, V. \& Bawendi, M.G. Color-selective photocurrent enhancement in coupled J-aggregate/nanowires formed in solution. Nano Lett. 11, 2655-2659 (2011).

17. Bredas, J.L., Norton, J.E., Cornil, J. \& Coropceanu, V. Molecular understanding of organic solar cells: the challenges. Acc. Chem. Res. 42, 1691-1699 (2009).

18. Wurthner, F. \& Meerholz, K. Systems chemistry approach in organic photovoltaics. Chem.-Eur. J. 16, 9366-9373 (2010).

19. Palmer, L.C. \& Stupp, S.I. Molecular self-assembly into one-dimensional nanostructures. Acc. Chem. Res. 41, 1674-1684 (2008).

20. Shao, H., Nguyen, T., Romano, N.C., Modarelli, D.A. \& Parquette, J.R. Self-assembly of 1-D n-type nanostructures based on naphthalene diimide-appended dipeptides. $J$. Am. Chem. Soc. 131, 16374-16376 (2009).

21. Balakrishnan, K. et al. Nanobelt self-assembly from an organic n-type semiconductor: propoxyethyl-PTCDI. J. Am. Chem. Soc. 127, 10496-10497 (2005).

22. Tovar, J.D. Supramolecular construction of optoelectronic biomaterials. Acc. Chem. Res. 46, 1527-1537 (2013).

23. Babu, S.S., Praveen, V.K. \& Ajayaghosh, A. Functional pi-gelators and their applications. Chem. Rev. 114, 1973-2129 (2014).

24. Gorl, D., Zhang, X., Stepanenko, V. \& Wurthner, F. Supramolecular block copolymers by kinetically controlled co-self-assembly of planar and core-twisted perylene bisimides. Nat. Commun. 6, 7006 (2015).

25. Gemayel, M.E. et al. Tuning the photoresponse in organic field-Effect transistors. J. Am. Chem. Soc. 134, 2429-2433 (2012). 
26. Sagade, A.A. et al. High-mobility field effect transistors based on supramolecular charge transfer nanofibres. Adv. Mater. 25, 559-564 (2013).

27. Hollamby, M.J. et al. Directed assembly of optoelectronically active alkyl-pi-conjugated molecules by adding n-alkanes or pi-conjugated species. Nature Chem. 6, 690-696 (2014).

28. Zhang, Y. et al. Organic single-crystalline $\mathrm{p}-\mathrm{n}$ junction nanoribbons. J. Am. Chem. Soc. 132, $11580-11584$ (2010).

29. Cui, Q.H. et al. Coaxial organic p-n heterojunction nanowire arrays: one-step synthesis and photoelectric properties. Adv. Mater. 24, 2332-2336 (2012).

30. Zhang, W. et al. Supramolecular linear heterojunction composed of graphite-like semiconducting nanotubular segments. Science 334, 340-343 (2011).

31. Faramarzi, V. et al. Light-triggered self-construction of supramolecular organic nanowires as metallic interconnects. Nature Chem. 4, 485-490 (2012).

32. Moulin, E., Cid, J.J. \& Giuseppone, N. Advances in supramolecular electronics - from randomly self-assembled nanostructures to addressable self-organized interconnects. $A d v$. Mater. 25, 477-487 (2013).

33. Kim, B.J., Yu, H., Oh, J.H., Kang, M.S. \& Cho, J.H. Electrical transport through single nanowires of dialkyl perylene diimide. J. Phys. Chem. C 117, 10743-10749 (2013).

34. Hulteen, J.C. \& Vanduyne, R.P. Nanosphere lithography - a materials general fabrication process for periodic particle array surfaces. J. Vac. Sci. Technol. A 13, 1553-1558 (1995).

35. Sinitskii, A. \& Tour, J.M. Patterning graphene through the self-assembled templates: toward periodic two-dimensional graphene nanostructures with semiconductor properties. J. Am. Chem. Soc. 132, 14730-14732 (2010).

36. Gao, T.C., Wang, B.M., Ding, B., Lee, J.K. \& Leu, P.W. Uniform and ordered copper nanomeshes by microsphere lithography for transparent electrodes. Nano Lett. 14, 2105-2110 (2014).

37. Gao, P.Q. et al. Large-area nanosphere self-assembly by a micro-propulsive injection method for high throughput periodic surface nanotexturing. Nano Lett. 15, 4591-4598 (2015).

38. Bao, Z. et al. Toward controllable self-assembly of microstructures: selective 
functionalization and fabrication of patterned spheres. Chem. Mater. 14, 24-26 (2002).

39. Cao, Q. et al. Arrays of single-walled carbon nanotubes with full surface coverage for high-performance electronics. Nature Nanotech. 8, 180-186 (2013).

40. Dintinger, J., Klein, S. \& Ebbesen, T.W. Molecule-surface plasmon interactions in hole arrays: Enhanced absorption, refractive index changes, and all-optical switching. $A d v$. Mater. 18, 1267-1270 (2006).

41. Parker, I.D. \& Kim, H.H. Fabrication of polymer light-emitting-diodes using doped silicon electrodes. Appl. Phys. Lett. 64, 1774-1776 (1994).

42. Baeg, K.J., Binda, M., Natali, D., Caironi, M. \& Noh, Y.Y. Organic light detectors: photodiodes and phototransistors. Adv. Mater. 25, 4267-4295 (2013).

43. Yu, H., Bao, Z.A. \& Oh, J.H. High-performance phototransistors based on single-crystalline $\mathrm{n}$-channel organic nanowires and photogenerated charge-carrier behaviors. Adv. Funct. Mater. 23, 629-639 (2013).

44. Levermore, P.A., Jin, R., Wang, X.H., de Mello, J.C. \& Bradley, D.D.C. Organic light-emitting diodes based on poly(9,9-dioctylfluorene-co-bithiophene) (F8T2). Adv. Funct. Mater. 19, 950-957 (2009).

45. Lei, T., Dou, J.H. \& Pei, J. Influence of alkyl chain branching positions on the hole mobilities of polymer thin-film transistors. Adv. Mater. 24, 6457-6461 (2012).

46. Li, Y.F. Molecular design of photovoltaic materials for polymer solar cells: toward suitable electronic energy levels and broad absorption. Acc. Chem. Res. 45, 723-733 (2012).

47. Shaw, P.E., Ruseckas, A. \& Samuel, I.D.W. Exciton diffusion measurements in poly(3-hexylthiophene). Adv. Mater. 20, 3516-3520 (2008).

48. Najafov, H., Lee, B., Zhou, Q., Feldman, L.C. \& Podzorov, V. Observation of long-range exciton diffusion in highly ordered organic semiconductors. Nature Mater. 9, 938-943 (2010).

49. Pinto, R.M. et al. Effect of molecular stacking on exciton diffusion in crystalline organic semiconductors. J. Am. Chem. Soc. 137, 7104-7110 (2015).

50. Sung, J., Kim, P., Fimmel, B., Wurthner, F. \& Kim, D. Direct observation of ultrafast coherent exciton dynamics in helical pi-stacks of self-assembled perylene bisimides. Nat. 
Commun. 6, 8646 (2015).

\section{Acknowledgements}

This work was financially supported by EC through the ERC project SUPRAFUNCTION (GA-257305) and the Marie Curie ITN project iSwitch (GA No. 642196), the ANR Equipex Union (ANR-10-EQPX-52-01), the Labex projects CSC (ANR-10-LABX-0026 CSC) and NIE (ANR-11-LABX-0058 NIE) within the Investissement d'Avenir program ANR-10-IDEX-0002-02, and the International Center for Frontier Research in Chemistry (icFRC).

\section{Author contributions}

L.Z., E.O. and P.S. conceived the experiment and designed the study: L.Z. performed the experiments and developed the fabrication method: X.L.Z. performed SEM characterization: E.P. and G.B. made transient response analysis: L.Z., E.O., S.L.L. and A.K. measured the optoelectronic devices: L.Z., E.O., T.W.E. and P.S. co-wrote the paper. All authors discussed results and contributed to the interpretation of data as well as contributed to editing the manuscript.

\section{Competing financial interests}

The authors declare no competing financial interests

\section{Additional information}

Supplementary information is available in the online version of the paper. Reprints and permission information is available online at www.nature.com/reprints. Correspondence and requests for materials should be addressed to P.S. or to E. O.. 
a

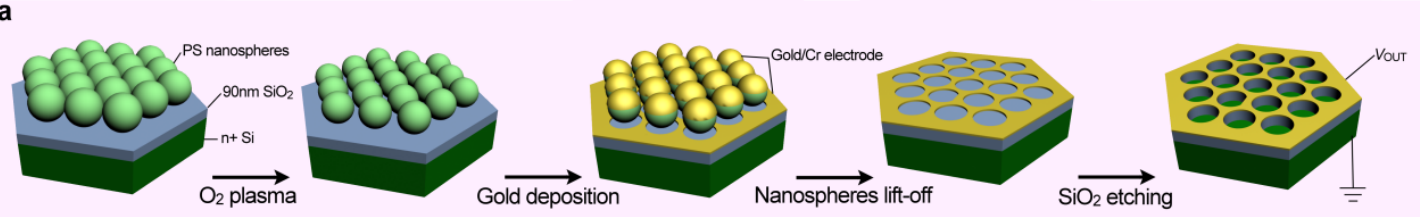

b
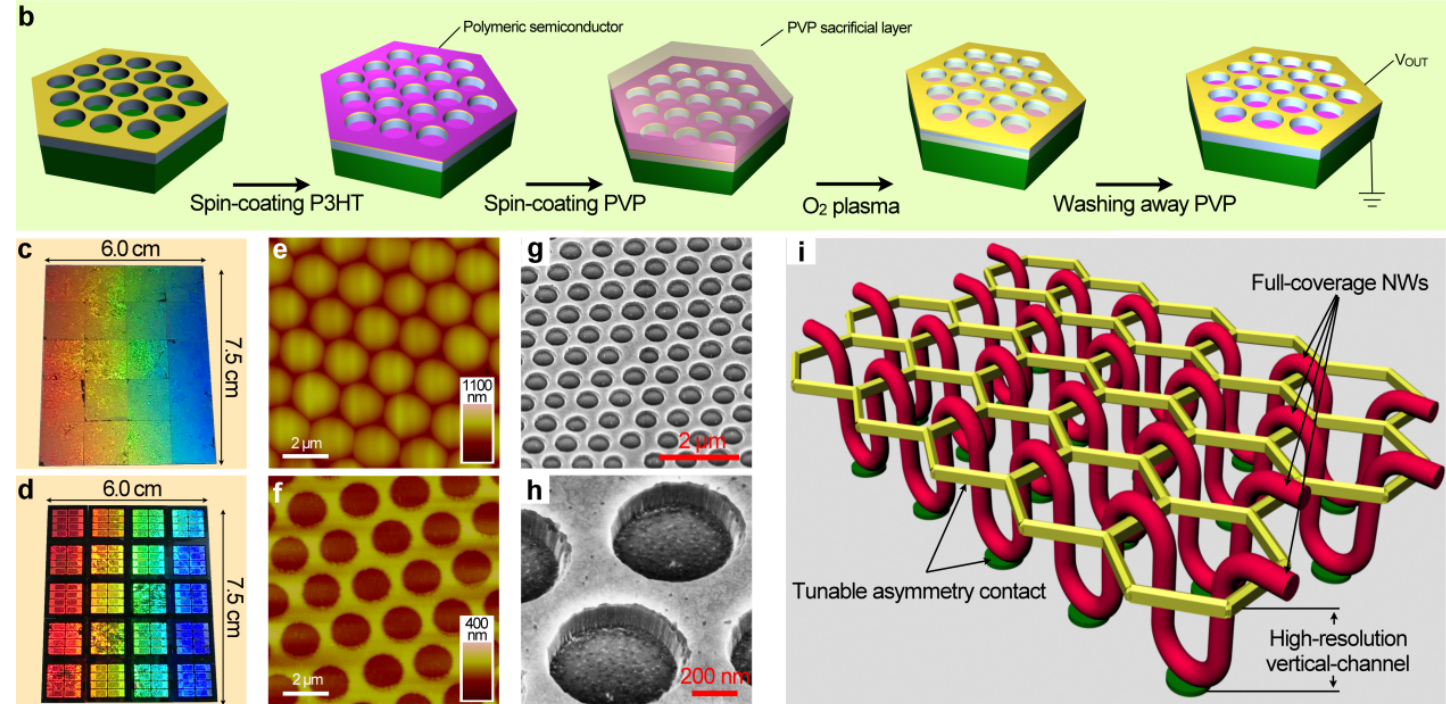

Figure 1| Design of vertical-channel nanomesh scaffold with asymmetrical and tunable

contact. a) Fabrication procedure of hexagonal hole-array nanomesh scaffold through nanospheres lithography and reactive ion etching. The geometry parameters of the resulting nanostructure could be tuned by PS nanospheres diameter, etching dose of $\mathrm{O}_{2}$ plasma and dielectric layer thickness on the wafer. b) Zone-selective modification of the bottom silicon contact with polymeric semiconductor like P3HT. c) Photograph of large-area PS nanospheres monolayer on silicon wafer, and d) resulting nanomesh electrodes after reactive ionic etching. e) AFM image (topography) of PS nanospheres monolayer, and f) final nanomesh scaffold. g) and $\mathbf{h}$ ) SEM images taken on bare nanomesh electrodes (substrate tilt angle $=54^{\circ}$ ). Top gold electrode and bottom silicon electrode is spaced by $90 \mathrm{~nm} \mathrm{SiO}_{2}$ layer. i) Cartoon showing high density nanowire photodiodes realized by vertical-channel nanomesh scaffold (dielectric layer and silicon substrate are omitted). 

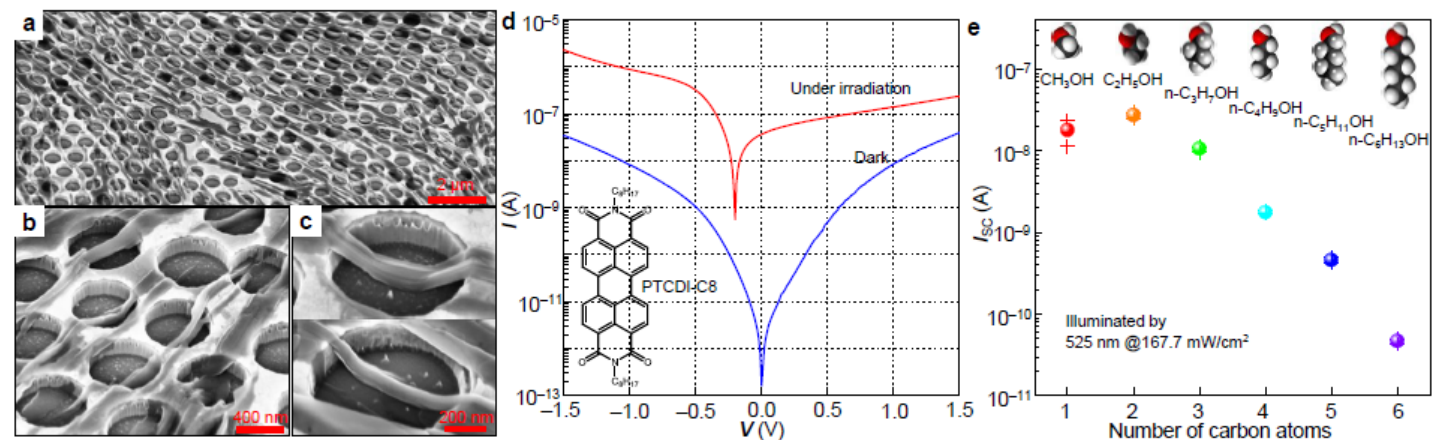

Figure 2| Photovoltaic effect of PTCDI-C8 supramolecular nanowires fully covering a

bare nanomesh scaffold. a), b) and c) SEM images taken on nanomesh electrodes bearing PTCDI-C8 nanowires, which was grown from ethanol non-solvent. The nanomesh scaffolds bearing single nanowire in c) were fabricated from PS nanospheres with different diameters, i.e. $800 \mathrm{~nm}$ PS nanospheres upper image and $1100 \mathrm{~nm}$ PS nanospheres lower image. It is clear that the nanowire starts to make conformal contact with the underlying electrode only at distance of ca. $150 \mathrm{~nm}$ from the hole edge, regardless of the hole diameter. d) Photovoltaic response of PTCDI-C8 supramolecular nanowires when illuminated by 470 nm blue light @ $212.9 \mathrm{~mW} / \mathrm{cm}^{2}$. The molecular structure of PTCDI-C8 is depicted in the inset. e) Short circuit current $\left(I_{\mathrm{SC}}\right)$ of photovoltaic cell with supramolecular nanowires produced from varying non-solvent from methanol to 1-hexanol. The round symbols represent the average $I_{\mathrm{SC}}$ measured on 6 devices and the error bars represent the highest and lowest current value measured in each case. The space-filling models and chemical formulae display all the alcohols used to grow PTCDI-C8 nanowires of different size and rigidity. 
Table 1| Photovoltaic property dependence on geometrical parameters

\begin{tabular}{ccccc}
\hline Samples: $^{\mathrm{a}}$ & \multicolumn{2}{c}{ Responsivity $^{\mathrm{b}}$} & \multicolumn{2}{c}{$V_{\mathrm{OC}}$} \\
\hline Sphere diameter & \multicolumn{2}{c}{$(R, \mu \mathrm{A} / \mathrm{W})$} & \multicolumn{2}{c}{$(\mathrm{V})$} \\
\hline & Methanol & Ethanol & Methanol & Ethanol \\
\hline $480 \mathrm{~nm}$ & 0.113 & 0.174 & 0.14 & 0.14 \\
\hline $600 \mathrm{~nm}$ & 0.393 & 0.717 & 0.18 & 0.19 \\
\hline $800 \mathrm{~nm}$ & 0.868 & 0.903 & 0.22 & 0.20 \\
\hline $1100 \mathrm{~nm}$ & 1.30 & 1.732 & 0.23 & 0.23 \\
\hline
\end{tabular}

${ }^{\mathrm{a}}$ Thermal treatment at $150{ }^{\circ} \mathrm{C}$ for 30 minutes; ${ }^{\mathrm{b}} R$ value is measured under $525 \mathrm{~nm}$ green light with the power density of $167.7 \mathrm{~mW} / \mathrm{cm}^{2}$. 

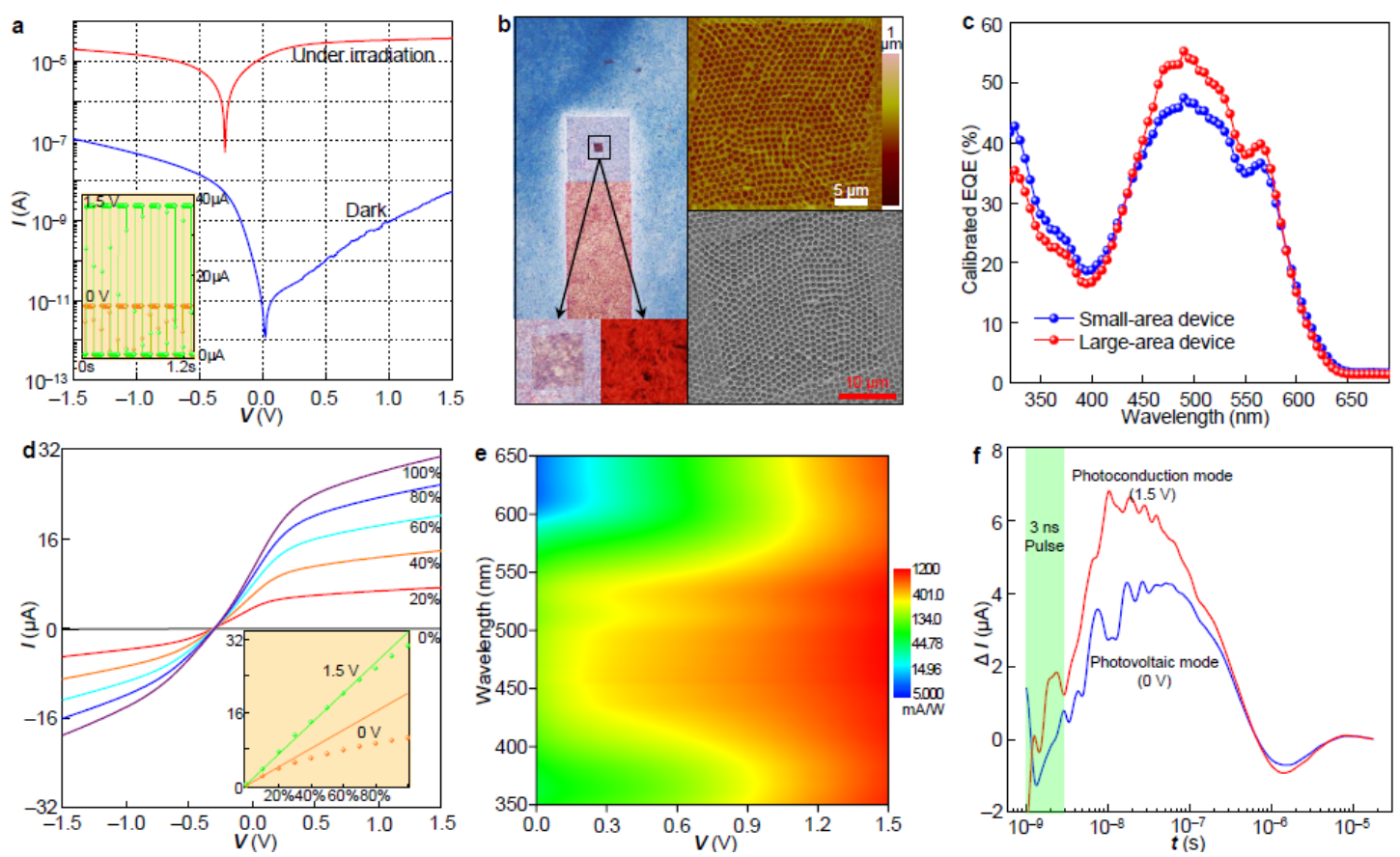

Figure 3| Photovoltaic effect of PTCDI-C8 supramolecular nanowires on P3HT modified

nanomesh scaffold. a) Electrical measurement with (blue line) and without (red line) light illumination@ @ $470 \mathrm{~nm}$ and $212.9 \mathrm{~mW} / \mathrm{cm}^{2}$. The device area is $0.07 \mathrm{~cm}^{2}$ and the bottom silicon electrode is modified by P3HT interlayer. Inset shows the photo-switching cycles driven by $0 \mathrm{~V}$ and $1.5 \mathrm{~V}$ working voltage. The signal-to-noise ratio could reach $10^{7}$ at $0 \mathrm{~V}$ and maintain $10^{4}$ at $1.5 \mathrm{~V}$. b) Illustration of small surface area nanowire photovoltaic device $(28 \times$ $28 \mu \mathrm{m}^{2}$ ). There are 697 holes bearing 1260 nanowires in the small region of $784 \mu^{2}$. c) Calibrated external quantum efficiency for both the large $\left(0.07 \mathrm{~cm}^{2}\right)$ and small area $\left(784 \mu \mathrm{m}^{2}\right)$ PTCDI-C8 supramolecular nanowires devices. After calibration, both the large area and small area device exhibit similar EQE value dependent on the irradiation wavelength. d) $I-V$ traces upon irradiation at different light intensity. The inset shows relationship between photocurrent and incident light power with $0 \mathrm{~V}$ and $1.5 \mathrm{~V}$ working voltage applied. The non-idealities in $I-V$ trace may suggest the presence of an un-optimized contact issue. The possible origins of such contact resistance are discussed in detail in Fig. S15. e) Calibrated responsivity of the small device at different driving voltage $(0 \mathrm{~V}$ to $1.5 \mathrm{~V})$ and varying wavelength $(350 \mathrm{~nm}$ to $650 \mathrm{~nm}$ ). The best $R$ at $0 \mathrm{~V}$ is $196 \mathrm{~mA} / \mathrm{W}$ (500 nm green light illumination) and it further increases to $1.2 \mathrm{~A} / \mathrm{W}$ when the nanowires device is biased at $1.5 \mathrm{~V}$. f) Time dependence of transient photocurrent response of P3HT/PTCDI-C8 nanowires devices to a $500 \mathrm{~nm}, 3 \mathrm{~ns}$ 
light pulse. The green shaded area indicates the laser pulse duration. [Laser pulse energy $\sim 20$ $\left.\mathrm{nJ} / \mathrm{cm}^{2}\right]$. The measurement protocol is thoroughly described in the method section. The negative current with the flow direction opposite to photocurrent peak originates from the interference brought by charge photo-generation and discharging process upon laser pulse at $\mathrm{Si} / \mathrm{SiO}_{2}$ and $\mathrm{Si} / \mathrm{P} 3 \mathrm{HT}$ interfaces. The negative signal could be reduced by using bare nanomesh without P3HT modification layer to remove Si/P3HT interface (Figure S16). Complete elimination of such effect would require to replace the silicon electrode with a metal one.
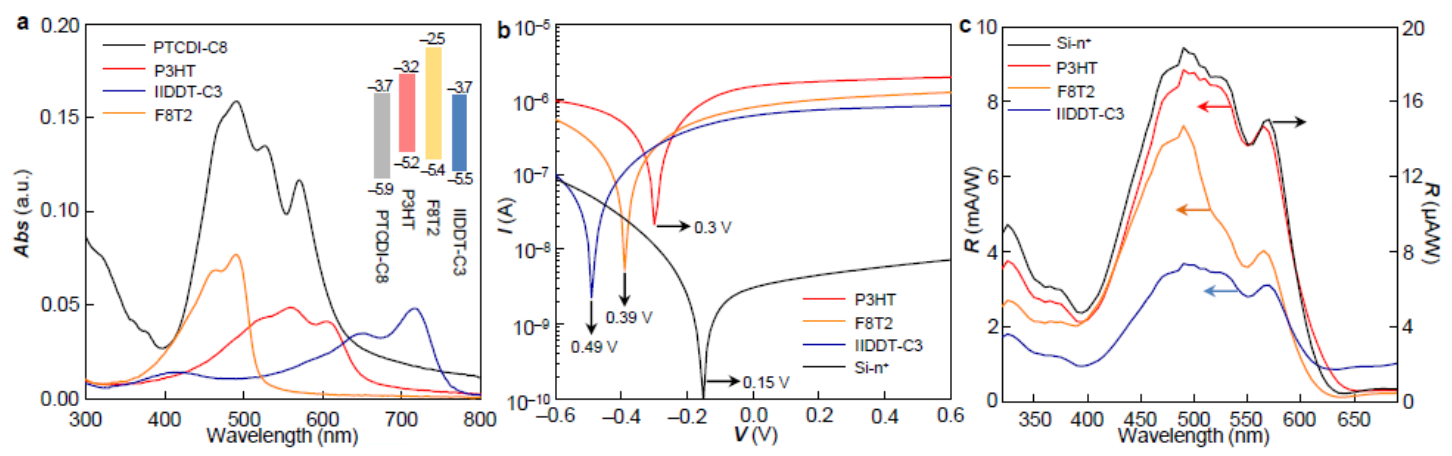

Figure 4| Device engineering with different hole transporting layers. a) UV-Vis absorbance of PTCDI-C8 nanowires and P3HT, F8T2, IIDDT-C3 thin films which are deposited on quartz using the same condition for photovoltaic device. The inset shows the HOMO-LUMO energy level alignment of these four organic semiconductors with the unit of eV. b) $I-V$ curves, and c) non-calibrated photo responsivity of supramolecular nanowires devices with bare, P3HT, IIDDT-C3 and F8T2 modified silicon anodes. The experimental results shown in panel b) were collected under $500 \mathrm{~nm}$ and $2.43 \mathrm{~mW} / \mathrm{cm}^{2}$ illumination. 
Table 2| Photovoltaic response of PTCDI-C8 supramolecular nanowires with different anode modification layer.

\begin{tabular}{ccccccc}
\hline Samples & $\mathrm{EQE}^{\mathrm{a}}$ & $V_{\mathrm{OC}}$ & $\mathrm{FF}$ & $\mathrm{PCE}^{\mathrm{a}}$ & $R^{\mathrm{a}}$ & $R^{\mathrm{b}}$ \\
\hline & & $(\mathrm{V})$ & & & $(\mathrm{mA} / \mathrm{W})$ & $(\mathrm{mA} / \mathrm{W})$ \\
\hline Si-n $^{+}$ & $0.11 \%$ & 0.15 & 0.36 & $0.0027 \%$ & 0.443 & 0.428 \\
\hline P3HT & $53.8 \%$ & 0.30 & 0.30 & $2.2 \%$ & 216.8 & 206.8 \\
\hline F8T2 & $41.6 \%$ & 0.39 & 0.29 & $2.1 \%$ & 167.6 & 117.2 \\
\hline IIDDT-C3 & $22.3 \%$ & 0.49 & 0.26 & $1.3 \%$ & 89.87 & 84.91 \\
\hline
\end{tabular}

${ }^{\mathrm{a}}$ The EQE, PCE and responsivity (R) are obtained by using $\mathrm{P}=2.43 \mathrm{~mW} / \mathrm{cm}^{2}$ at $\lambda=500 \mathrm{~nm}$.

${ }^{\mathrm{b}}$ Responsivity measured under $525 \mathrm{~nm}$ light illumination in order to minimize F8T2 contribution to the PTCDI-C8 nanowires photovoltaics. All the current related data were calibrated by considering a surface coverage factor of $4.05 \%$ in order to evaluate the intrinsic photovoltaic process in supramolecular nanowires.

\section{Method Section}

Materials. Polystyrene (PS) nanospheres mono-dispersed suspension $(10 \mathrm{w} \%$, in water), Poly(3-hexylthiophene-2,5-diyl)

$(\mathrm{M} n$ 54000-75000, $99.995 \%)$, $N, N^{\prime}$-Dioctyl-3,4,9,10-perylenedicarboximide (98\%), Poly(9,9-dioctylfluorene-alt-bithiophene) (99.9\%) and Poly(4-vinylphenol) (Mw 25000) were purchased from Sigma-Aldrich without further purification. The small bandgap p-type polymer of IIDDT-C3 were purchased from 1-Material and used as received. The silicon wafer was purchased from Fraunhofer with 90 -nm thick thermally grown $\mathrm{SiO}_{2}$ dielectric layer ( $n$-doping level $\sim 310^{17} \mathrm{~cm}^{-3}$ ).

Reactive ion etching conditions. The gold nanomesh electrode on top of $90 \mathrm{~nm} \mathrm{SiO}_{2}$ was patterned by nanospheres lithography, according to literature. ${ }^{35,36}$ Then we deposited a thin layer of $\operatorname{LiF}(\sim 20 \mathrm{~nm})$ by shadow mask to define the device surface. LiF was chosen as the 
resist for several reasons: i) it can be easily removable with water. ii) it is electrically inert. iii) It exhibit high corrosion stability in reactive ion etching. Using a Plasmalab 80 plus reactive ion etching system purchased from Oxford Instruments, the $\mathrm{SiO}_{2}$ dielectric layer not being protected by gold and LiF would be removed under a condition of $200 \mathrm{~W}$ plasma power and 5 sccm $\mathrm{C}_{2} \mathrm{~F}_{6} / 25 \mathrm{sccm}$ Ar mixed gas.

Anode modification. P3HT/chlorobenzene $(2.5 \mathrm{mg} / \mathrm{ml})$ and PVP/propylene glycol methyl ether acetate $(120 \mathrm{mg} / \mathrm{ml})$ were spin-coated onto the nanomesh scaffold in sequence. Before spin-coating PVP, the substrate periphery was cleaned by a cotton bud soaked in chloroform in order to improve wettability. Then oxygen plasma treatment was performed to selectively burn off the PVP/P3HT layer on top of nanomesh. The etching dose was verified by AFM characterization. The residual PVP could be washed away by ethanol. The electrical performances of all the modification layers, i.e., P3HT, F8T2 and IIDDT-C3, were explored by integrating them in bottom-contact bottom-gate OFET devices (Fig. S17; Fig. S18 and Table S2). The AFM topology analysis was supplied as Fig. S19.

Hierarchical assembling of full-coverage PTCDI-C8 supramolecular nanowires film. For the first step, we made $0.25 \mathrm{mg} / \mathrm{ml}$ PTCDI-C8 solution in chloroform. Then $200 \mu \mathrm{L}$ of PTCDI-C8 solution was injected into $3 \mathrm{ml}$ of alcohol solvent. Supramolecular nanowire formation occurs within seconds because molecular interactions are favoured in the non-solvent. The supramolecular nanowires were carefully collected and dispersed again in a solvent mixture of hexane/ethanol (3:1). The dispersion was dropped onto water surface to form floating nanowire film, which was compressed by $50 \mu \mathrm{L} \mathrm{PVP} /$ Ethyl acetate $(100 \mathrm{mg} / \mathrm{ml})$ before transferring the supramolecular nanowires onto the substrate. Although water is involved during device fabrication, we should note the moisture does not affect the electrical properties of hierarchically assembled PTCDI-C8 supramolecular nanowires, which exhibited typical n-type transfer curve in OFETs (Fig. S20). The on/off ratio could reach $10^{5}$ upon annealing at $80{ }^{\circ} \mathrm{C}$ inside $\mathrm{N}_{2}$ glovebox. The drain current would increase steadily after further annealing with elevated temperature from $150{ }^{\circ} \mathrm{C}$ to $225^{\circ} \mathrm{C}$ at a step of $25{ }^{\circ} \mathrm{C}$ due to the improved molecular ordering (Fig. S20).

Optoelectronic measurement. All the optoelectronic characterization was performed inside nitrogen filled glovebox. For the irradiation, we used Polychrome V as monochromatic source, 
which was purchased from Till Photonics. The output power spectrum has been calibrated by The PM100A Power Meter from Thorlab company and the data was supplied as Fig. S21. Coupled with monochromatic light source, Keithley 2636A system source meter was used to make electrical characterization.

Transient photocurrent measurements. Transient photocurrent measurements were performed by applying a DC voltage $\left(V_{\mathrm{b}}\right)$ ranging from $-1.5 \mathrm{~V}$ to $1.5 \mathrm{~V}$ between highly doped n-type Si substrate and top Au nanomesh (cathode). The Si substrate is referred as an anode and was connected to zero potential. The AC signal from the cathode, separated by a circuit comprising a capacitor and a coil (bias-T) was connected to a $2 \mathrm{GHz}$ current amplifier (the schematic of the measurement setup is shown in the inset of Fig. S22). The charge carriers were created by a pulsed laser impinging perpendicular to the top Au surface. The laser pulse duration was 3-4 ns, and its repetition rate was $10 \mathrm{~Hz}$. The samples were measured upon irradiation at $\lambda=500 \mathrm{~nm}$. The time dependence of the photocurrent $(I(\mathrm{t}))$ was recorded by a 2.5 GHz oscilloscope. The zero-time was set to the beginning of the laser pulse, which was determined from the intersection of the dark current and rising slope of the $I(\mathrm{t})$. Here $I(\mathrm{t})$ curve has been smoothed and background $I(\mathrm{t})$ from an empty nanomesh scaffold without supramolecular nanowires has been removed. All measurements were performed in a nitrogen atmosphere with $\mathrm{H}_{2} \mathrm{O}$ and $\mathrm{O}_{2}$ levels below $10 \mathrm{ppm}$. All devices were tested in terms of current-voltage characteristics prior to the transient photocurrent characterization. 